\title{
16
}

\section{Fuzzy logic for similarity analysis}

\author{
J. A. B. Montevechi and G. L. Torres \\ Escola Federal de Engenharia de Itajubá \\ CP 50 - Itajubá - MG - 37500-000, Brazil, tel.: +5535 629 1212, \\ fax: +5535629 1148, e-mail: arnaldo\%efei.uucp@dcc.ufmg.br \\ P. E. Miyagi and M. R. P. Barretto \\ Universidade de São Paulo - Escola Politécnica \\ CP: 61548 - São Paulo - SP - 05508-900, Brazil
}

\begin{abstract}
In this article a procedure for obtaining similarities using fuzzy logic is discribed. It permits taking into account uncertainties and ambiguities usually present in manufacturing. Aspects of the relational data base are presented which can aggregate design and manufacture information. How to assign membership to the features that will be analysed, the similarity analysis to the resemblance relation and part processing information are also presented. This procedure makes it possible the elaboration of one software which will be a interesting tool to the manufacture of small lots, it integrates the informations of design and manufacture and makes possible a rationalization of resources.
\end{abstract}

\section{Keywords}

Group Technology, part family, fuzzy logic, membership attribution

\section{INTRODUCTION}

Most papers about part family formation assume that information about cost and processing time, demand of part, etc.. are accurate. It is usually supposed that a part only belongs to one family. Nevertheless, in many cases it does not occur. The analysis of grouping, making use of fuzzy logic, can provide a solution to this problem. However, few articles have been published dealing with the problem of uncertainty, the formation of manufacturing cells and 
the part families. Likewise, those articles consider such questions isolately neglecting the development of methods to be shared by all company users (Montevechi, 1994).

The part similarities, which is the basic aspect for family formation, consist of a close classification in geometry, function, material and /or process. It may be not sufficient to describe part features using yes or no labels, when accurate classification is required (Montevechi, 1994). In order to obtain an efficient and flexible classification which considers uncertainties, thus eliminating the shortcoming of the currently employed methods, this article describes a procedure that makes use of fuzzy logic for the part family formation. Fuzzy membership function permits taking into account the inherent uncertainties into part features description. Thus producing more realistic results. The use of this technique, will make the part family formation more sensible. The membership value, which lies between 0 and 1 can express what extension of the feature the part has. The closer the value is to 1 , more quantity of feature the part has.

First are described details of a relational data. The grouping principle employed is also described which consists of choosing one threshold value to the similarity. Once this threshold value is chosen, two elements will be in the same group if, for example, in case of similarity function, the similarity between them is greater than the threshold value. Since the similarity relationship is not necessarily transitive, it is necessary to employ the fuzzy matrix theory to form the closest structure which permits the separating data in exclusive and separated groups which are, in essence, an equivalent class over a certain threshold value.

For process similarity a procedure is shown to search information of similarities that should guide the formation of manufacturing cells.

Another important aspect described is the possibility to use qualitative data, such as, complex, easy, hard, high surface roughness, etc.... How to translate this information into numerical values, which is essential to similarity analysis, is also shown.

The object of this methodology, is to develop an alternative procedure to traditional methods for obtaining similarity. To this purpose, it is necessary to integrate apropriate approachs that can incorporate the uncertainty which nowadays serve isolated aspects of similarity analysis.

\section{DATA BASE}

An extremely important component of the proposed methodology to obtain the part families, is the data base providing the features that will be classified according to their similarities. In the data base all important information about several component features of the company have to be available, including design and manufacturing features. However, it should be borne in mind that no coding procedure is proposed, which is common in the Classification and Codification System (CCS). The part input information is made according to discription of its features. This fact is very important because it permits data handling by all company user, fact that does not happen with most CCS. Features that may be in the data base are shown in Figure 1.

Development a relational data base permits adding more data and features. It is not possible in CCS, because after the coding is obtained the insertion of new features will be difficult. 


\begin{tabular}{|l|l|l|l|}
\hline \multicolumn{2}{|c|}{ DESIGN ATTRIBUTES } & \multicolumn{2}{c|}{ MANUFACTURE ATTRIBUTES } \\
\hline External shape & Main dimensions & Main process & Machine tool \\
\hline Internal shape & Material & Largest dimension & Lead time \\
\hline Tolerances & Superficial Finishing & Superficial Finishing & Lot size \\
\hline
\end{tabular}

Figure 1 Example of data base design and manufacturing features.

\section{MEMBERSHIP ATTRIBUTION}

The parts for the classification by their similarities is represented in a $\mathbf{n} \times \mathbf{~ m}$ matrix form (part $\mathrm{x}$ feature). This matrix will be formed with the data base features important for the grouping analysis. Basically in the data base it is possible to have two types of features, namely quantitative and qualitative.

Since the features can be quantitative or qualitative, it is important to develop a procedure for the grouping which can deal with these two kinds of features in a unified way. It is necessary to transfer the data to these features in the same unit. Otherwise, there will be a scale problem. In this methodology the data of different features are expressed by memberships. To this purpose, each part feature is given a membership between 0 and 1 , which will put an end to the scale problem.

Membership values of quantitative features can be expressed directly in function of values obtained from the data base. For example, the length feature whose values for 7 parts are given by the vector $[10.00 ; 8.5 ; 5.5 ; 3.75 ; 6.25 ; 8.00 ; 7.50]$, the membership values for this feature, can be calculated by dividing each length value by the largest value of the vector (i.e. 10.00). This procedure results in another vector, that is the membership vector, given by $[1.00 ; 0.850 ; 0.55 ; 0.375 ; 0.625 ; 0.800 ; 0.750]$. These values also can be given by any of the expressions suggested by Xu and Wang (1989).

Likewise, it is possible to use graphs with a more suitable membership function, which automatically gives the values of membership to the selected features.

With qualitative features, now, the attribution of membership is not so easy. How to transform a qualitative information, such as part complexity or with high roughtness in a number?

To solve this problem, such as proposed by Arieh and Triantaphyllou (1992), it is possible to utilize the AHP (Analytic Hierarchy Process) method (Saaty, 1977), which by means of a comparative analysis permits the calculation of membership for qualitative attributes. These comparative analysis are made in pairs between the attributes of the feature in question. It is necessary to make a matrix of comparison for the attributes for each feature. Features are, for example, roughness, shape complexity, length, etc, whereas the attributes are the feature designation as small, large, complex, little complex, high roughness, etc. Saaty proposed that to provide attribute comparison should be used values from the finite set: $\{1 / 9,1 / 8, \ldots, 1,2 \ldots, 8,9\}$. These matrices are calculated by the evaluation of the importance of an attribute over the other, using an appropriate scale (Saaty, 1977).

Each entry of the matrix is a pair of judgment. After the matrix of comparison is defined, the eigenvalue $\left(\lambda_{\max }\right)$ and its respective eigenvector will be calculated. The eigenvector will represent the memberships that can be used for the attributes in question and the eigenvalue is 
the measure or rate of consistency of the result.

To illustrate this method, one of the features which may be important for the obtaining similarity is the complexity of shape evaluated by a analist. The attributes of this feature originating from the data base range from very complex, to complex, mean complexity, low complexidade and very low complexity. All the parts, of the data base, have one of these qualitative values for its feature of complexity. By means of the scale of priority shown, a specialist will provide the matrix A of Figure 2.

\begin{tabular}{|c|c|c|c|c|c|}
\hline & very complex & complex & $\begin{array}{c}\text { mean } \\
\text { complexity }\end{array}$ & $\begin{array}{c}\text { low } \\
\text { complexity }\end{array}$ & $\begin{array}{c}\text { very low } \\
\text { complexity }\end{array}$ \\
\hline much complex & 1 & 3 & 5 & 7 & 9 \\
\hline complex & $1 / 3$ & 1 & 3 & 5 & 7 \\
\hline mean complexity & $1 / 5$ & $1 / 3$ & 1 & 3 & 5 \\
\hline low complexity & $1 / 7$ & $1 / 5$ & $1 / 3$ & 1 & 3 \\
\hline very low complexity & $1 / 9$ & $1 / 7$ & $1 / 5$ & $1 / 3$ & 1 \\
\hline
\end{tabular}

Figure 2 Matrix A of comparison by pairs for the feature shape complexity.

In matrix $\mathbf{A}$ of comparison, entry $\mathrm{a}_{\mathrm{ij}}$ indicates the number that estimates the relative membership of attribute $\mathbf{A}_{\mathbf{i}}$ when it is compared with the attribute $\mathbf{A}_{\mathbf{j}}$. Obviously, $\mathrm{a}_{\mathrm{ij}}=1 / \mathrm{a}_{\mathrm{j}}$. With the eigenvector, the memberships that can be used for the similarity classification are available, obviously after testing the consistence of the result to conclude that the answer is good (Saaty, 1977). In this case, the memberships (one of eigenvectors normalized for the greatest weight to be equal 1), after the calculation, for the attributes values will be to very complex: 1, complex:0.517, mean complexity: 0.254 , low complexity: 0.125 and very low complexity: 0.064 . These values of memberships now will give a weight for each of the parts from the data base. These memberships represent the importance of complexity of shape for each part.

After the memberships for the several features (qualitative and quantitative) are calculated, now, it is necessary to have a procedure to obtain the clusters of similar parts.

\section{ANALYSIS OF SIMILARITY FOR THE FEATURES}

A principle that can be used for the grouping is to choose a threshold value for the similarity. Once this threshold value is chosen, two elements will be in the same grouping if the similarity between them is larger than the value of comparison.

To estimate the resemblance between pairs of data, it is possible to use the convention of arranging the data in form of matrix. Each entry in this matrix will represent the proximity between two parts. This relationship, called $\mathbf{S}$ which is a matrix $\mathbf{n} \times \mathbf{n}$, represents the similarity between different parts. To obtain this matrix it is possible to utilize several formulaes for the calculation of similarity, as examplified by the expression (1).

The symmetric matrix can be used directly in the analysis of fuzzy grouping. The similarity of parts consist of a very close classification in geometry, function, material and /or process. The similarity as seen, will be obtained throught the membership manipulation. 


$$
S\left(x_{i}, x_{j}\right)=\frac{\sum_{k=1}^{p} \min \left(\mu_{k}\left(x_{i k}\right), \mu_{k}\left(x_{j k}\right)\right)}{\frac{1}{2} \sum_{k=1}^{p}\left(\mu_{k}\left(x_{i k}\right)+\mu_{k}\left(x_{j k}\right)\right)}
$$

But the matrix does not have the propriety of being transitive. Here what occurs is that there is a relationship of resemblance. Then, the following conclusion, that if $\mathbf{A}$ is similar to $\mathbf{B}$, and $\mathbf{B}$ is similar to $\mathbf{C}$, then $\mathbf{A}$ is similar to $\mathbf{C}$ will not be possible. To deal with this problem, it is necessary to transform the matrix of similarity into a transitive matrix. It is possible to use the fuzzy theory to transform this matrix (Montevechi, 1994). The transitive matrix is the matrix fuzzy equivalent.

Finally, given one $\propto$ level, the groupings of similar parts are obtained for the level chosen. With different $\propto$ values, different classifications will appear. The greater is the $\propto$ value, less parts will be classified in each family, thus more families will be formed.

An example of decomposition, for obtaining the families can be better understood through Figures 3 and 4. Figure 3 shows the attribution of some $\propto$ values and Figure 4 shows, for each one of the $(\propto)$ levels, the groupings formed. For example, for $\propto=0,9$, there are three groupings, the first one consisting of parts $\mathbf{A}, \mathbf{D}$ and $\mathbf{E}$, the second one, of part $\mathbf{B}$ and the third one, of part $\mathbf{C}$.

$\left.\mathrm{R}=\left[\begin{array}{ccccc}1 & 0,8 & 0,7 & 1 & 0,9 \\ 0,8 & 1 & 0,7 & 0,8 & 0,8 \\ 0,7 & 0,7 & 1 & 0,7 & 0,7 \\ 1 & 0,8 & 0,7 & 1 & 0,9 \\ 0,9 & 0,8 & 0,7 & 0,9 & 1\end{array}\right]=\operatorname{co,7} \cdot\left[\begin{array}{lllll}1 & 1 & 1 & 1 & 1 \\ 1 & 1 & 1 & 1 & 1 \\ 1 & 1 & 1 & 1 & 1 \\ 1 & 1 & 1 & 1 & 1 \\ 1 & 1 & 1 & 1 & 1\end{array}\right], 0,8 \cdot\left[\begin{array}{lllll}1 & 1 & 0 & 1 & 1 \\ 1 & 1 & 0 & 1 & 1 \\ 0 & 0 & 1 & 0 & 0 \\ 1 & 1 & 0 & 1 & 1 \\ 1 & 1 & 0 & 1 & 1\end{array}\right],\left[\begin{array}{lllll}1 & 0 & 0 & 1 & 1 \\ 0 & 1 & 0 & 0 & 0 \\ 0 & 0 & 1 & 0 & 0 \\ 1 & 0 & 0 & 1 & 1 \\ 1 & 0 & 0 & 1 & 1\end{array}\right],\left[\begin{array}{lllll}1 & 0 & 0 & 1 & 0 \\ 0 & 1 & 0 & 0 & 0 \\ 0 & 0 & 1 & 0 & 0 \\ 1 & 0 & 0 & 1 & 0 \\ 0 & 0 & 0 & 0 & 1\end{array}\right]\right)$

Figure 3 Decomposition of a similarity relationship.

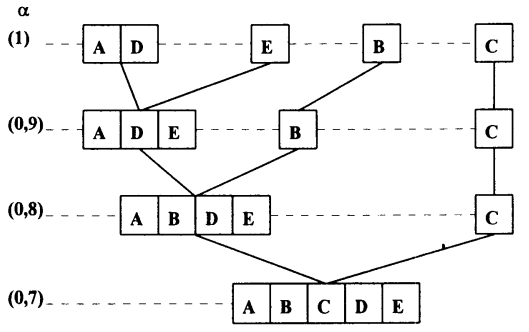

Figure 4 Tree shaped decomposition.

\section{ANALYSIS OF PROCESS SIMILARITY}

A problem that may happen, in the formulation for obtaining the similarity seen in the last item, is that the information about cell formation may not be enough. To prevent this from 
happening, it is possible to think in such a way to obtain the matrix of membership between part $\mathbf{x}$ machine, where the vagueness will also be considered. In this way an algorithm for checking the grouping of machines may be used.

If $\mathbf{n}$ parts and $\mathbf{m}$ machines are being considered to obtain manufacturing cells, usually the representation of the machines that processes each part is given by a binary matrix, where de entries are 1 or 0 . Due to the inflexibility of this matrix, another matrix should be developed, which should be called of nonbinary, represented by (2).

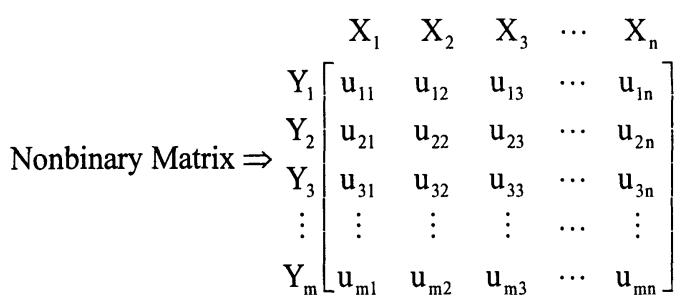

in (2), $X_{j}$ is a part and $j=1,2,3, \ldots, n$; $Y_{i}$ is a machine and $i=1,2,3, \ldots, m$ and $u_{i j}$ represents the relationship between part $\mathbf{j}$ and machine $\mathbf{i}$. In (2) it is possible to observe the property (3).

$$
0 \leq \mathrm{u}_{\mathrm{ij}} \leq 1 \text { for } \mathrm{i}=1,2,3, \ldots, \mathrm{m} ; \mathrm{j}=1,2,3, \ldots, \mathrm{n}
$$

The property defined by (3) indicates the intensity with which a machine is designated to process a determined part, a number near of 1 meaning a great potentiality to process the part, while with a number near 0 , the machine would definitely not be appropriate.

The elements of matrix (2) are calculated from mixed functions between machines and components, which is an interesting proposition from Zhang and Wang (1992).

To illustrate this procedure, for a small example, if the pertinence functions are those of Figure 5, for a hypothetic case of machine 1 of $\mathbf{m}$ available, for each one of the 7 parts that are to be grouped, with the $\mathbf{x}$ value (from a data base) for the feature in question, it is possible to do a chart of Figure 6.

In the same way that the values of Figure 6 are given, the process should be repeated for all the machines that will be analyzed, thus $\mathbf{m}$ matrices analogous to this is obtained. The minimum values of each row of Figure 6 result the vector \{machine $1\left[\begin{array}{lllllll}1 & 0 & 1 & 0.8 & 0 & 0.1 & 0.9\end{array}\right]$, which expresses the membership for the 7 parts of machine 1 .If the process for the $\mathbf{m}$ machine is repeated, $\mathbf{m}$ vectors will be calculated, which will consist of the nonbinary matrix that should be studied for obtaining the similarities of the process. If for the 7 parts example a universe of 7 machines is available, after the execution of the procedure, a nonbinary matrix as (4) may be obtained. The matrix (4) is the one that should be analyzed to have a solution of similarity of process.

Obtained the non-binary matrix, it is now necessary to run a proper grouping algorithm to get the possible manufacturing cells. Zhang and Wang (1992) shows the use of the Rank Order Clustering (ROC) (King, 1980) to analyze matrix (4). The result for this matrix is that cell 1 is composed of machines $Y_{1}, Y_{6}$ and $Y_{5}$ and family 1 of part $X_{3}, X_{1}, X_{7}$ and $X_{4}$, cell 2 is composed of machines $\mathrm{Y}_{7}, \mathrm{Y}_{2}, \mathrm{Y}_{3}$ and $\mathrm{Y}_{4}$ and family 2 of part $\mathrm{X}_{2}, \mathrm{X}_{5}$ and $\mathrm{X}_{6}$. 

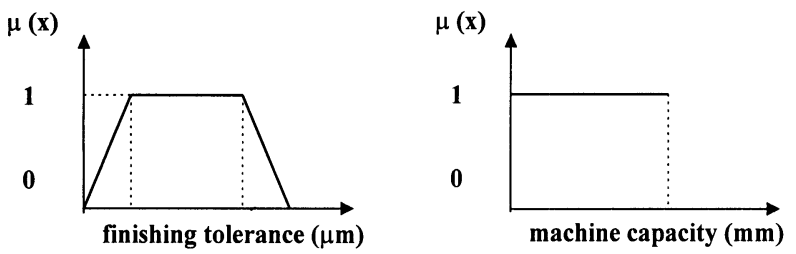

Figure 5 Example of membership function relative to machine 1.

\begin{tabular}{|c|c|c|}
\hline Parts & finishing tolerance & machine capacity \\
\hline 1 & 1 & 1 \\
\hline 2 & 1 & 0 \\
\hline 3 & 1 & 1 \\
\hline 4 & 0.8 & 1 \\
\hline 5 & 1 & 0 \\
\hline 6 & 0.1 & 1 \\
\hline 7 & 0.9 & 1 \\
\hline
\end{tabular}

Figure 6 Memberships given to each pair part $\mathrm{x}$ feature for the machine 1.

\begin{tabular}{|c|c|c|c|c|c|c|c|}
\hline & $X_{1}$ & $X_{2}$ & $\mathrm{X}_{3}$ & $\mathrm{X}_{4}$ & $X_{5}$ & $x_{6}$ & $X_{7}$ \\
\hline $\mathrm{Y}_{1}$ & 1 & 0 & 1 & 0.8 & 0 & 0.1 & 0.9 \\
\hline $\mathrm{Y}_{2}$ & 0 & 1 & 0.3 & 0 & 0.7 & 0.8 & 0 \\
\hline $\mathrm{Y}_{3}$ & 0.3 & 0.7 & 0 & 0 & 0.8 & 0.8 & 0 \\
\hline $\mathrm{Y}_{4}$ & 0 & 0.7 & 0 & 0.3 & 0.7 & 0 & 0.3 \\
\hline $\mathrm{Y}_{5}$ & 0.6 & 0.1 & 0.5 & 0 & 0 & 0 & 0.5 \\
\hline $\mathrm{Y}_{6}$ & 0.7 & 0.2 & 0.8 & 0.8 & 0.3 & 0 & 0.7 \\
\hline $\mathrm{Y}$ & 0 & 1 & 0.5 & 0 & 0.8 & 0.9 & 0 \\
\hline
\end{tabular}

The importance of utilizing the nonbinary matrix is that, after the grouping of machines is obtained, there is now the possibility of analyzing the machines that are more appropriate to process the part families. Furthermore, it makes sense to eliminate the machines that process similar operations. This characteristic is not possible if the binary matrix is used.

Other algorithms for the grouping can be adapted so that the nonbinary matrix will be used for the cell formation.

\section{CONCLUSIONS}

This paper is the synthesis of what is possible to do with fuzzy logic in order to deal with the problem of obtaining similarities, an important aspect for part families formation, considering the uncertainty present in the manufacturing environment. This procedure groups techniques that can cope with the problem of similarities isolately, and more comprehensively, making 
use of the same data base, a fact that usually is necessary, although it is not possible in the most currents tools.

With the procedure described here it is possible to provide a new contribution for the Group Technology.

The development of this model can also supply a solution for the problem of setup time decrease, once it is possible to retrieve information of process and geometry similarities together. In this way, is possible to profit by these similarities so that the preparation time of machines will be shorter. It is possible to observe then that with the rationalization, which is possible with the identification of similarities, work is being done to save the company resources. Finally, this methodology may be transformed into a software, which will be an interesting tool to the manufacture small lots. This consists of a different proposition as an alternative to current methods available.

\section{REFERENCES}

Arieh, D.B. and Triantaphyllou, E. (1992) Quantifying data for group technology with weighted fuzzy features. Int. J. Prod. Res, 30, 1285 - 1299.

King, J.R. (1980) Machine-component grouping in production flow analysis: an approach using a rank order clustering algorithm. Int. J. Prod. Res. , 18, 213 - 232.

Montevechi, J.A.B. (1994) Formação de famílias de peças prismáticas utilizando lógica Fuzzy. Doctorate Qualifying Examination, São Paulo, EP-USP.

Saaty, T. L. (1977) A Scaling Method for Priorities in Hierarchical Structures. Journal of Mathematical Psychology, 15, 234 - 281.

$\mathrm{Xu}, \mathrm{H}$. e Wang, H.P (1989) Part family formation for GT applications based on fuzzy mathematics. Int. J. of Prod. Res., 27, 1637 - 1651.

Zhang, C. and Wang, H.P (1992) Concurrent Formation of Part Families and Machine Cells Based on the Fuzzy Set Theory. Journal of Manufacturing Systems, 11, 61 - 67.

\section{BIOGRAPHY}

J.A.B. Montevechi is Assistent Professor, received the master degree from Florianópolis University, Brazil, in 1989. Research areas include: Group Technology, expert systems and fuzzy logic.

G.L. Torres is Full Professor, received the degree of $\mathrm{Ph} . \mathrm{D}$. from École Polytechnique, Canada, in 1990. Research areas include: expert systems, fuzzy logic and neural networks to power systems problems.

P.E. Miyagi is Associate Professor, received the degree of Dr.Eng. from Tokyo Institute of Technology, Japan, in 1988. In 1994 received the degree of L.Doc. Research areas include: discrete event, dynamic systems, sequential control and systems programming.

M.R.P. Barretto is Assistent Professor, received the degree of Dr.Eng. from University of São Paulo, Brazil, in 1993. Research areas include: production planning and control, production order programming. 